\title{
What's New in the Clinical Management of Disorders of Social Cognition?
}

\begin{abstract}
Social cognition refers to the ability to use social cues to infer the meaning and intentions behind the behaviour of others in order to respond in a socially adaptive manner. It is increasingly recognised that disorders of social cognition, including problems with emotion perception, theory of mind, conversational inference, morality judgements, decision making and social inhibition, characterise many developmental and psychiatric disorders and are highly relevant to many with acquired brain injuries or diseases, especially the frontotemporal dementias. This review provides an introduction and overview of the papers in this special edition on social cognition and places these in the context of other recent research. In doing so, several current issues in the clinical management of social cognition are delineated. Given that social cognition seems to be a sensitive predictor of psychosocial function, the assessment of social cognition is seen by many clinicians to be important although which profession is responsible is yet to be resolved. Issues in how social cognition are assessed are discussed, including the importance of context to social cognition, its interactive nature and the need to recognise influences such as family upbringing, gender and emotional state on social cognitive performance. There also needs to be development of tests that address all aspects of social cognition, including decision making and inhibition. Finally, this review discusses intervention research. Interventions are especially well developed in relation to schizophrenia, and less well developed in brain injury. These appear to be generally effective although treatments for emotional self-awareness are yet to be developed.
\end{abstract}

Keywords: brain injuries, dementia, schizophrenia, theory of mind, social cognition, emotion perception, assessment, intervention

There has been a seismic shift in clinical understanding of social cognition over the past two decades. The idea that social cognition is separate to non-social cognition and can be independently affected is a relatively recent concept although descriptors of people with brain damage with normal cognition, but poor social skills have been in the literature for a long time. EVR, for example, was a highly intelligent individual who suffered extensive resection of orbit medial frontal cortex due to removal of a meningioma (Eslinger $\&$ Damasio, 1985). As a result, his cognitive skills on conventional tests remained superior, but his social conduct deteriorated suggestive of primary impairment of the social self.

Even so, it is only relatively recently that social cognition has come under systematic examination.
One of the clearest, most cogent accounts of the unique aspects of social cognition was first provided by Penn, Corrigan, Bentall, Racenstein and Newman (1997). They argued that, as schizophrenia was essentially an interpersonal disorder, the problems experienced by people with schizophrenia must be conceptualised as problems of social cognition. Further, while non-social cognitive impairments are likely to contribute to difficulties in the social realm, they argued that the perception and interpretation of social information could not simply be understood as a subset of general cognitive ability. They pointed out that social cognition requires the ability to make inferences about unobservable matters (such as thoughts), and to deal with mutable information that is personally relevant rather than neutral, objective and stable and 
likely to cause its own reaction in the observer and change as a result.

Since that time, there has been a groundswell of interest in social cognition and recognition of the pivotal role that it plays in community integration. Clinicians who work with people with brain disorders, whether these are developmental, psychiatric or due to acquired injury or disease, are only too aware that social and emotional impairments are a major obstacle to successful psychosocial functioning. Social cognition impairments not only characterise disorders of schizophrenia, but are also seen as the defining characteristic of Autism Spectrum Disorders (American Psychiatric Association, 2013) and manifest in other intellectual and genetic developmental disabilities (Roelofs, Wingbermühle, Kessels, \& Egger, 2017). In the field of traumatic brain injury (TBI), $84 \%$ of clinicians reported that disorders of social cognition affect at least half of their clients (Kelly, McDonald, \& Frith, 2016). Social cognitive disorders are also diagnostic of specific dementias, notably fronto-temporal dementias (Hazelton, Irish, Hodges, Piguet, \& Kumfor, 2017; Kipps, Nestor, Acosta-Cabronero, Arnold, \& Hodges, 2009; Lough et al., 2006).

\section{Defining the Sub-components of Social Cognition}

As a result of this increased interest in social cognition, the nature of its inter-related components is rapidly being delineated. At base, there is general agreement that social cognition refers to the ability to use social cues to infer the meaning and intentions behind the behaviour of others in order to respond in a socially adaptive manner (McDonald, 2013). Typically, social cognition is thought to include emotion perception, that is, the ability to recognise the emotional states of others from facial, vocal and body cues, and Theory of Mind (ToM), which is the ability to judge what another person may be thinking and the intentions behind their behaviour. ToM is critical to understanding everyday conversational pragmatic inferences such as sarcasm, hyperbole, hints, etc. Social cognition also includes empathy that refers to the capacity to 'put oneself in another's shoes', to see things from their point of view (cognitive empathy), or to share their feelings (affective empathy). To be able to do this, one must also be self-aware, able to distinguish between feelings for others and one's own feelings. Attributional bias, which refers to the personal filter through which the behaviour of others is interpreted (such as seeing ambiguous social behaviour as hostile) has been associated with schizophrenia (Savla, Vella, Armstrong, Penn, \& Twamley, 2013) but is also relevant to other conditions such as brain injury (Cassels, McDonald, Kelly, \& Togher, 2016). Other facets of social cognition such as the development of social knowledge and morality are particularly relevant to children in whom maturational processes are complicated by the presence of brain injury, as discussed in this volume (Chiasson, Elkaim, Well, Crevier, \& Beauchamp, 2017). Social cognition also impinges on behaviour such as the capacity to make effective decisions to enhance prosocial engagement (Adlam, Adams, Turnbull, Yeates, \& Gracey, 2017) while inhibiting antisocial urges (Honan, Allen, Fisher, Osborne-Crowley, \& McDonald, 2017).

\section{Using Social Cognition to Predict Outcomes}

Now that we are becoming better equipped to describe and identify problems in social cognition, we can use this information to better predict psychosocial outcomes. Indeed, social cognitive abilities have been shown to be better able to predict community function than non-social cognitive variables in people with schizophrenia (Fett et al., 2011). In adults with TBIs, problems recognising emotions in others have been shown to be predictive of poor communication skills and use of humour (McDonald et al., 2013; McDonald, Flanagan, Martin, \& Saunders, 2004; Milders, Ietswaart, Crawford, \& Currie, 2008; Watts \& Douglas, 2006) and poor social integration (Knox \& Douglas, 2009).

Children with brain disorders are especially vulnerable to disorders of social cognition as these skills underpin the maturation of skills needed for increasingly complex social interactions through childhood and adolescence (Yeates et al., 2007). Long-term social outcomes following paediatric TBI are predicted by poor executive function, social information processing and pragmatic language skills (Yeates et al., 2004). Further, while poor social outcomes have been frequently attributed to severe, diffuse injuries such as TBI, in this volume, Chiasson et al. (2017) found that 15 children with focal brain lesions had impaired social cognitive skills, specifically moral reasoning, decision making and empathy. They also found these children experienced sociobehavioural problems suggesting a possible link between the two.

Changes in social cognition have been found to predict psychosocial function in dementia although this varies according to the nature of dementia profile. Loss of empathy predicts carer burden in semantic dementia and loss of a caring relationship in 
the behavioural variant of FTD (Hsieh, Irish, Daveson, Hodges, \& Piguet, 2013). As reported in this volume, loss of affective empathy in people with primary progressive aphasic disorders is also associated with increased carer burden (Hazelton et al., 2017). Interesting, while people with Alzheimer's disease (AD) also experience changes to social cognition, there is less evidence that this affects the quality of their relationships. Poveda, Laidlaw, Macleod, Power, \& Osborne-Crowley (2017) report that while their group of adults with AD suffered problems with social cognition as assessed by The Awareness of Social Inference Test (TASIT), this was not related to either carer distress or relationship continuity. It may be that deficits on performance-based social cognition measures in $\mathrm{AD}$ are less indicative of reduced socio-emotional functioning than the impact of non-social cognitive impairments, especially in the earlier stages of the disease. This may be why the correlation with carer relationships is not evident. Indeed, in a separate study, when people with $\mathrm{AD}$ were rated by their carers for their levels of empathy, they were found to have significantly higher cognitive and affective empathy than people with FTD. Further, empathy ratings did not predict carer burden in $\mathrm{AD}$ but did in FTD (Hsieh et al., 2013).

\section{Social Cognition and the Psychosocial Context: A Two Way Street?}

Overall, these studies show that, by focusing on social cognition, more nuanced relationships with psychosocial outcomes are revealed. This is a welcome advance given that assessments of (nonsocial) cognitive abilities in clinical groups have had only modest ability to predict everyday functioning (Odhuba, van den Broek, \& Johns, 2005; Silver, 2000). Of course, it is also important to consider that factors other than the clinical disorder itself may impact upon social cognition. This is especially true when considering children, who are developing their social cognitive skills in the context of an injury or other disorder (Chiasson et al., 2017; Ryan et al., 2017). Ryan et al.(2017) present data on children with mild to severe TBI in which they found that severity of injury did not predict poor social cognition but family emotional responsivity was strongly associated. Thus, the nature of the family context and the emotional and interpersonal milieu in which the child is raised makes an important contribution to their social cognitive development independent of brain injury. There are numerous other factors that may interact with social cognitive skills that need to be considered in both children and adults at risk of social cognitive disorders. For example, mood disorders are associated with disorders of social cognition (George et al., 1998; Lahera et al., 2013), are highly prevalent in neurological conditions and may exacerbate or compound social cognitive impairments arising from brain injury (McDonald, Fisher, \& Flanagan, 2015).

Another factor that is often overlooked in social cognitive studies is that there is significant inter-individual variation in social cognitive skills that does not, necessarily, parallel non-social cognitive abilities. Importantly, unlike non-social cognition, there are gender differences in social cognition that are apparent from early childhood (Dadds et al., 2009) through adolescence (McDonald et al., 2015) with a female advantage for recognition of emotions from face and voice (Collignon et al., 2010) and self-reported empathy (Baron-Cohen et al., 2015). As reported in this volume, this advantage appears to maintain even following brain injury (Zupan, Babbage, Neumann, \& Willer, 2017) and needs to be considered when evaluating levels of social cognitive impairment.

\section{Issues in the Clinical Assessment of Social Cognition}

The assessment of non-social cognition has a long tradition. Clinicians have access to standardised tests that have been extensively researched and normed and, in many cases, translated across languages. They are also well defined according to which profession has responsibility for the particular domain (language $=$ speech pathology, memory = neuropsychology, etc.). This is not the case for the relatively new area of social cognition. While there are tools available from the clinical and experimental literature, assessment of social cognition is not the norm. Indeed, as reported by Kelly, Frith, \& McDonald (2017) in this volume, less than $16 \%$ of clinicians report using any kind of formal assessment of social cognition.

Further, there appears to be differences within professions as to who is held responsible for the assessment of social cognition with clinical psychologists, clinical neuropsychologists, occupational therapists and speech pathologists each believing the responsibility primarily lies with the other (Kelly et al., 2016). This is probably inevitable given that social cognition is a new domain that crosses boundaries between perception, executive function, emotional processing, personality, behaviour, language and everyday function. The coming decade will be interesting to see who claims social cognition within their professional realm of expertise. 


\section{Assessing Emotion Perception, ToM and Pragmatic Inference}

A major similarity between social cognition and non-social cognition is that many attributes, such as emotion perception, ToM, recognition of pragmatic inferences (which rely on inferring the intention of speakers), moral judgements etc., can be assessed with performative tasks with associated normative data. There is huge scope to develop such tests. My own test, TASIT (McDonald, Flanagan, Rollins, \& Kinch, 2003), which uses audiovisual vignettes of actors, has proven to be useful in a range of clinical disorders including TBI, schizophrenia, right hemisphere damage, autism spectrum disorders and frontotemporal dementia (McDonald, 2012). However, it only assesses specific aspects of social cognition, namely emotion perception, ToM and conversational inference, and specifically as these pertains to adults and adolescents. These aspects of social cognition are the most commonly assessed. For example, several other batteries such as the Florida Affect Battery (Bowers, Blonder, \& Heilman, 1991) and the Diagnostic Analysis of Non-Verbal Accuracy (Nowicki, 2010) also assess emotion perception and are backed up with normative data. The child assessment battery NEPSY-II (Korkman, Kirk, \& Kemp, 2007) assesses both emotion and ToM in children from 3 to16 years. Some speech pathology language assessments such as the Social Language Development Test (Bowers, Huisingh, \& LoGiudice, 2010) and the Clinical Evaluation of Language Fundamentals (Wiig \& Secord, 2014) assess conversational and pragmatic inference from child to early adulthood.

One question for assessment, even of these well-established constructs, is whether performance as assessed using de-contextualised social stimuli, is actually indicative of real-life judgements in situations where far more cues are typically present. Turkstra and colleagues (2017) in this volume examine this issue with respect to emotion recognition. Reassuringly, they found that de-contextualised facial expressions (as typically presented) were recognised as accurately as those placed in context in both normal healthy adults and people with brain injury. However, they also found that when allowed to respond in a free format rather than multiple choice, at least $50 \%$ of responses could not be categorised as one of the six 'basic' emotions (happy, sad, angry, disgusted, fearful and surprised) supposedly depicted by the stimuli and that people with TBI gave quite different responses. This raises important questions about how we should be assessing emotion perception.
Another criticism of established social cognition tests is that they usually require making third person judgements, for example, passively watching a scene and judging what the people in the scene are doing and thinking about each other. In contrast, we are usually participants in the social contexts in which we make social judgements. Some of these judgements will require interpreting social cues that are directed towards ourselves. One way to enhance the interactive nature of social cognitive assessments is the use of virtual reality. Canty, Neumann, Fleming and Shum (2015) developed such a measure where actors in videoed contexts talk directly to camera, giving a first person quality to the interactions. This test that measures both cognitive and affective mental judgements proved to be sensitive to social functioning and holds great promise as a clinical assessment instrument.

\section{Assessing Morality, Decision Making and Social Inhibition}

For other aspects of social cognition there are, in fact, very few clinical instruments. As a significant advance, in this volume, Chiasson and colleagues (2017) describe a social moral assessment instrument that has proven sensitive to brain injury in children and is useful for determining whether children have reached appropriate developmental stages in their moral reasoning. As with the instrument devised by Canty et al. (2015), the The Socio-Moral Reasoning Aptitude Level Task (SoMoral: Beauchamp, Dooley, \& Anderson, 2013) uses visual vignettes from a first person perspective to increase participant engagement. Furthermore, So-Moral has been adapted into an iPad version, PEERS, which is specifically for clinical use and near completion with 500+ child norms already collected (Anderson, personal communication). The translation of experimental social cognitive tasks into clinical tools is a very welcome advance. It is not always, however, a straightforward process as is apparent in efforts to find a good measure of social decision making. Decision making in socio-emotional contexts has been assessed in the past using the Iowa Gambling Test (IGT) (Bechara, Damasio, Tranel, \& Damasio, 2005). This card based gambling task purports to tap into 'somatic markers', whereby individuals are guided by their own physiological responses. These responses are triggered by anticipation of reward or punishment in complex situations and may index learning even before the individual is consciously aware of the contingency (Bechara, Damasio, Tranel, \& Damasio, 1997). The IGT is a complex test and its practical use in clinical 
assessment is questionable. In this volume, Adlam and colleagues (2017) test the clinical utility of a shorter, simpler card-based gambling test, the Bangor Gambling Task and find that this, also, has problems associated with its sensitivity to decision making impairment. Another option is to assess decision making in a clearly social context. Kelly, McDonald and Kellet (2014) developed the Social Decision Making Test (SDMT) where, rather than winning at cards, the aim was to be included by others in a virtual 'ball game'. The SDMT was sensitive to brain injury where the IGT was not, so this may be the way forward in developing tasks of social decision making.

Another important facet of social cognition is the ability to inhibit socially undesirable responses for those that are more likely to enhance social interactions. There are a number of measures of inhibition within the neuropsychological domain such as the Haylings Sentence Completion Test (Burgess \& Shallice, 1997) and various Stroop type tasks. Once again, however, these lack a social context so whether they are likely to be sensitive to specific loss of social inhibition is unclear. One approach with which to address this dilemma was taken by Osborne-Crowley, McDonald and Francis (2016) using an interview format in which participants were asked open questions such as "tell me about an embarrassing moment you have had'. Independent raters identified people with brain injury as having greater self-disclosure and disinhibition than controls and this correlated with relative's reports of disinhibited behaviour indicating good validity. It was not associated with traditional neuropsychological measures of inhibition, strengthening the argument that these constructs may be different (Osborne-Crowley et al., 2016). The drawback is this measure is not easy to use clinically as it requires two raters to achieve good reliability.

An alternative approach has been developed by Honan et al. (2017) and reported in this volume. Modelled on the Haylings Sentence Completion test, the Social Disinhibition Test (SDT) uses pictures of people engaged in awkward social situations and requires participants to describe characters in the scenes under three conditions: without restriction; with the requirement that nothing negative is stated and with requirement that something positive is stated. Scores for items range from 0 to 2 . People with brain injury had difficulty especially when required not to be negative. A relationship was found between the SDT and the Haylings test as well as TASIT, suggesting it taps into disinhibition as measured more broadly and also social cognition. Disappointingly, the relationship with informant rated loss of (emotional) control was low. This may reflect the fact that emotional loss of control is different to verbal disinhibition, but further research is needed to determine this.

\section{Using Questionnaires to Measure Social Cognition}

Performative tests of social cognition can be supplemented by self or informant-report questionnaires. For some attributes of social cognition, notable empathy, self-report questionnaires are highly appropriate as empathy is essentially a subjective state. While there are concern that many people with brain injury may have limited insight and unable to complete questionnaires reliably, research has demonstrated that this need not be the case (Kinsella, Moran, Ford \& Ponsford, 1988). Numerous studies have used self-report empathy questionnaires such as the Balanced Emotional Empathy Scale (Mehrabian, 2000), the Interpersonal Reactivity Index (IRI: Davis, 1983) and the Empathy Quotient (EQ: Baron-Cohen \& Wheelwright, 2004) to report deficits in empathy following brain injury (de Sousa et al., 2011; Neumann, Zupan, Malec, \& Hammond, 2013; Rushby et al., 2016; Wood \& Williams, 2008) and also Autism Spectrum Disorders (Baron-Cohen et al., 2015).

If it is unlikely that the person can complete a questionnaire reliably, a close informant can provide a reasonable assessment of empathic behaviour. A number of studies have used the IRI filled in by a close relative to examine loss of empathy in people with dementia. Using this, loss of empathy has been found in the frontotemporal dementias, including the behavioural variant (Dermody et al., 2016) and semantic dementia, but not AD (Rankin, Kramer, \& Miller, 2005). Further, in this volume, Hazelton et al. (2017) demonstrate that people with dementia characterised as non-fluent primary progressive aphasia, i.e., both progressive non-fluent aphasia and logopenic progressive aphasia also experience reduced cognitive and affective empathy.

\section{The Push for Rehabilitation of Social Cognition}

Rehabilitation of social cognitive deficits has a relatively long tradition, especially in schizophrenia and to a lesser extent developmental conditions such as Autism Spectrum Disorders. In contrast, there is relatively little remediation research for social cognition impairments in people with acquired brain injuries (see Cassels et al. (2016) for review). The primary focus of social cognitive 
remediation has been to improve skills in emotion perception, ToM and attributional bias. Treatment has been conducted with a range of approaches from a singular treatment focused on a particular skill to more comprehensive approaches targeting two or more such skills and possibly also non-social cognition. As reported in this volume, Roelofs Wingbermühle, Egger, \& Kessels (2017) conducted a meta-analysis of 41 studies reporting on treatment of social cognitive disorders in over 1500 patients with acquired brain disorders or neuropsychiatric symptoms. Encouragingly, they found that targeted treatments were especially effective, particularly for emotion perception, ToM and social perception (the ability to use social cues to determine social roles, rules, etc). Comprehensive and broad based interventions were effective also, and had impact upon social functioning.

This meta-analysis also attempted to include alexithymia, which is a disorder in emotional selfawareness, however, no studies met inclusion criteria. Self-awareness is an important ingredient in social cognition, because to have empathy, one must be able to both share feelings with another while recognising that one's own feelings are separate. Indeed, it is increasingly thought that much of social cognition relies upon the ability to simulate the experiences of others in oneself (Decety \& Grèzes, 2006), with the attendant need to know the difference between the two. In general, there are few studies that have attempted to address emotional self-awareness in neuropsychiatric and neurological disorders, although remediation of self-awareness more generally has been the subject of research in acquired brain injury (Ownsworth, Fleming, Desbois, Strong, \& Kuipers, 2006; Ownsworth, McFarland, \& Young, 2000). In this volume, Lamberts, Fasotti, Boelen and Spikman (2017) report on a treatment trial that used self-monitoring to improve executive function. They found that self-awareness was improved as a result. Importantly, emotion perception was significantly correlated to self-awareness pre-treatment. This accord with the view that selfawareness is a central construct to social cognition. It also raises the important point that social cognitive deficits may present an obstacle to any rehabilitation, if the treatment relies heavily on social interaction with the therapists and other members of the treatment group.

\section{Conclusion}

In conclusion, social cognition is emerging as a highly relevant target for clinical management in a wide range of neurological, developmental and psychiatric disorders. Because it is a relatively new domain of cognition, clinicians are yet to decide who will take responsibility for its assessment and management. New tests are needed that take into account the special features of social cognition, including the interactive nature of the relationship between the observer and his/her social context and inherent differences in social cognitive ability reflecting individual differences in gender, family upbringing, mood and potentially many other social-emotional influences. There is good reason to be optimistic that interventions can be effective for social cognition, but new research is needed to apply these to people with acquired brain injury, and to really consider the importance of selfawareness in social cognitive ability. The papers in this volume represent exciting new initiatives looking at each of these topics.

\section{References}

Adlam, A.-L.R., Adams, M., Turnbull, O., Yeates, G., \& Gracey, F. (2017). The bangor gambling task: Characterising the performance of survivors of traumatic brain injury $18(1), 62-73$.

Baron-Cohen, S., Bowen, D.C., Holt, R.J., Allison, C., Auyeung, B., Lombardo, M.V., ... Lai, M.-C. (2015). The 'reading the mind in the eyes' test: Complete absence of typical sex difference in $\sim 400$ men and women with autism. PLoS One, 10(8), ArtID e0136521.

Baron-Cohen, S., \& Wheelwright, S. (2004). The empathy quotient: An investigation of adults with asperger syndrome or high functioning Autism, and normal sex differences. Journal of Autism and Developmental Disorders, 34(2), 163-175. doi: 10.1023/b:jadd.0000022607.19833.00.

Beauchamp, M.H., Dooley, J.J., \& Anderson, V. (2013). A preliminary investigation of moral reasoning and empathy after traumatic brain injury in adolescents. Brain Injury, 27, 896-902.

Bechara, A., Damasio, H., Tranel, D., \& Damasio, A. R. (1997). Deciding advantageously before knowing the advantageous strategy. Science, 275(5304), 1293-1294.

Bechara, A., Damasio, H., Tranel, D., \& Damasio, A. R. (2005). The Iowa gambling task and the somatic marker hypothesis: Some questions and answers. Trends in Cognitive Sciences, 9(4), 159-162.

Bowers, D., Blonder, L.X., \& Heilman, K.M. (1991). Florida affect battery. Gainsville, FL: Centre for Neuropsychological Studies, University of Florida.

Bowers, L., Huisingh, R., \& LoGiudice, C. (2010). Social language development test-adolescent manual. East Moline, IL: LinguiSystems, Inc.

Burgess, P.W., \& Shallice, T. (1997). The hayling and brixton tests. San Antonio: Pearson PsychCorp Assessment.

Canty, A.L., Neumann, D.L., Fleming, J., \& Shum, D. H. (2015). Evaluation of a newly developed measure 
of theory of mind: The virtual assessment of mentalising ability. Neuropsychological Rehabilitation, 1-37. doi: 10.1080/09602011.2015.1052820.

Cassels, A., McDonald, S., Kelly, M., \& Togher, L. (2016). Learning from the minds of others: A review of social cognition treatments and their relevance to traumatic brain injury. Neuropsychological Rehabilitation. Retrieved from: https://doi.10.1080/ 09602011.2016.1257435

Chiasson, V., Elkaim, L., Well, A., Crevier, L., \& Beauchamp, M. (2017). Moral reasoning in children with focal brain insults to frontotemporal regions. Brain Impairment, 18(1), 102-116.

Collignon, O., Girard, S., Gosselin, F., Saint-Amour, D., Lepore, F., \& Lassonde, M. (2010). Women process multisensory emotion expressions more efficiently than men. Neuropsychologia, 48(1), 220-222. Retrieved from: http://doi.org/10.1016/j. neuropsychologia.2009.09.007

Dadds, M.R., Hawes, D.J., Frost, A.D.J., Vassallo, S., Bunn, P., Hunter, K., \& Merz, S. (2009). Learning to 'talk the talk': The relationship of psychopathic traits to deficits in empathy across childhood. Journal of Child Psychology and Psychiatry, 50(5), 599-606. doi: 10.1111/j.1469-7610.2008.02058.x.

Davis, M.H. (1983). Measuring individual differences in empathy: Evidence for a multidimensional approach. Journal of Personality and Social Psychology, 44(1), 113-126. doi: 10.1037/0022-3514.44.1.113.

de Sousa, A., McDonald, S., Rushby, J., Li, S., Dimoska, A., \& James, C. (2011). Understanding deficits in empathy after traumatic brain injury: The role of affective responsivity. Cortex, 47(5), 526-535. doi: 10.1016/j.cortex.2010.02.004.

Decety, J., \& Grèzes, J. (2006). The power of simulation: Imagining one's own and other's behavior. Brain Research, 1079, 4-14.

Dermody, N., Wong, S., Ahmed, R., Piguet, O., Hodges, J. R., \& Irish, M. (2016). Uncovering the neural bases of cognitive and affective empathy deficits in Alzheimer's disease and the behavioral-variant of frontotemporal dementia. Journal of Alzheimer's Disease, 53(3), 801-806. doi: 10.3233/JAD-160175.

Eslinger, P.J., \& Damasio, A.R. (1985). Severe disturbance of higher cognitive function after bilateral frontal ablation: Patient EVR. Neurology, 35, 17311741.

Fett, A.J., Viechtbauer, W., Dominguez, M., Penn, D.L., Van Os, J., \& Krabbendam, L. (2011). The relationship between neurocognition and social cognition with functional outcomes in schizophrenia: A metaanalysis. Neuroscience and Biobehavioral Reviews, 35, 573-588.

George, M.S., Huggins, T., McDermut, W., Parekh, P. I., Rubinow, D., \& Post, R.M. (1998). Abnormal facial emotion recognition in depression: Serial testing in an ultra-rapid-cycling patient. Behavior Modification, 22(2), 192-204.

Hazelton, J.L., Irish, M., Hodges, J.R., Piguet, O., \& Kumfor, F. (2017). Cognitive and affective empathy disruption in non-fluent primary progressive aphasia syndromes. Brain Impairment, 18(1), 117-129.

Honan, C.A., Allen, S.K., Fisher, A., Osborne-Crowley, K., \& McDonald, S. (2017). Social disinhibition: Piloting a new clinical measure in individuals with traumatic brain injury. Brain Impairment, 18(1), 74 87.

Hsieh, S., Irish, M., Daveson, N., Hodges, J.R., \& Piguet, O. (2013). When one loses empathy: Its effect on carers of patients with dementia. Journal of Geriatric Psychiatry and Neurology, 26(3), 174-184.

Kelly, M., Frith, M., \& McDonald, S. (2017). Assessment and rehabilitation of social cognition impairment after brain injury: Surveying practices of clinicians. Brain Impairment, 18(1), 11-35.

Kelly, M., McDonald, S., \& Frith, M. (2016). A survey of clinicians working in brain injury rehabilitation: Are social cognition impairments on the radar? Journal of Head Trauma Rehabilitation. doi: 10.1097/HTR.0000000000000269.

Kelly, M., McDonald, S., \& Kellet, D. (2014). Development of a novel task for investigating decision making in a social context following traumatic brain injury. Journal of Clinical and Experimental Neuropsychology, 36(9), 897-913.

Kinsella, G., Moran, C., Ford, B., \& Ponsford, J. (1988). Emotional disorder and its assessment within the severe head-injured population. Psychological Medicine, 18, 57-63.

Kipps, C.M., Nestor, P.J., Acosta-Cabronero, J., Arnold, R., \& Hodges, J.R. (2009). Understanding social dysfunction in the behavioural variant of frontotemporal dementia: The role of emotion and sarcasm processing. Brain, 132, 592-603.

Knox, L., \& Douglas, J. (2009). Long-term ability to interpret facial expression after traumatic brain injury and its relation to social integration. Brain and Cognition, 69, 442-449.

Korkman, M., Kirk, U., \& Kemp, S. (2007). NEPSY (2nd ed). New York: Pearson Assessment.

Lahera, G., Benito, A., Montes, J., Fernandez-Liria, A., Olbert, C., \& Penn, D. (2013). Social cognition and interaction training (SCIT) for outpatients with bipolar disorder. Journal of Affective Disorders, 146(1), 132-136.

Lamberts, K.F., Fasotti, L., Boelen, D.H.E., \& Spikman, J. M. (2017). Self-awareness after brain injury; relation with emotion recognition and effects of treatment. Brain Impairment, 18(1), 130-137.

Lough, S., Kipps, C.M., Treise, C., Watson, P., Blair, J. R., \& Hodges, J.R. (2006). Social reasoning, emotion and empathy in frontotemporal dementia. $\mathrm{Neu}$ ropsychologia, 44(6), 950-958.

McDonald, S. (2012). New frontiers in neuropsychological assessment: Assessing social perception using a standardised instrument, the awareness of social inference test. Australian Psychologist, 47(1), 39-48. doi: 10.1111/j.1742-9544.2011.00054.x.

McDonald, S. (2013). Impairments in social cognition following severe traumatic brain injury. Journal of 
the International Neuropsychological Society, 19, 231-246.

McDonald, S., English, T., Randal, R., Longman, T., Togher, L., \& Tate, R.L. (2013). Assessing social cognition and pragmatic language in adolescents with traumatic brain injuries. Journal of the International Neuropsychological Society, 19(5), 528538.

McDonald, S., Fisher, A., \& Flanagan, S. (2015). When diplomacy fails: Difficulty understanding hints following severe traumatic brain injury. Aphasiology. doi: 10.1080/02687038.2015.1070948.

McDonald, S., Fisher, A., Togher, L., Tate, R., Rushby, J., English, T., ... Francis, H. (2015). Adolescent performance on the awareness of social inference test: TASIT. Brain Impairment, 16(1), 3-18.

McDonald, S., Flanagan, S., Martin, I., \& Saunders, C. (2004). The ecological validity of TASIT: A test of social perception. Neuropsychological Rehabilitation, 14, 285-302.

McDonald, S., Flanagan, S., Rollins, J., \& Kinch, J. (2003). TASIT: A new clinical tool for assessing social perception after traumatic brain injury. Journal of Head Trauma Rehabilitation, 18, 219238.

Mehrabian, A. (2000). Manual for the balanced emotional empathy scale (BEES). Available from Albert Mehrabian, 1130 Alta Mesa Road, Monterey, CA 93940.

Milders, M., Ietswaart, M., Crawford, J.R., \& Currie, D. (2008). Social behavior following traumatic brain injury and its association with emotion recognition, understanding of intentions, and cognitive flexibility. Journal of the International Neuropsychological Society, 14, 318-326. doi: 10.10170S1355617708080351.

Neumann, D., Zupan, B., Malec, J.F., \& Hammond, F. (2013). Relationships between alexithymia, affect recognition, and empathy after traumatic brain injury. Journal of Head Trauma Rehabilitation, 29(1), E18-E27. doi: 10.1097/HTR.0b013e31827fb0b5.

Nowicki, S. (2010). Manual for the receptive tests of the diagnostic analysis of nonverbal accuracy 2 (DANVA2). Atlanta, GA: Dr Stephen Nowicki, Jr., Department of Psychology, Emory University, Retrieved from: snowick@emory.edu.

Odhuba, R.A., van den Broek, M.D., \& Johns, L.C. (2005). Ecological validity of measures of executive functioning. British Journal of Clinical Psychology, 44(2), 269-278.

Osborne-Crowley, K., McDonald, S., \& Francis, H. (2016). Development of an observational measure of social disinhibition after traumatic brain injury. Journal of Clinical and Experimental Neuropsychology, 38(3), 341-353. doi: 10.1080/13803395.2015.1115824.

Ownsworth, T., Fleming, J., Desbois, J., Strong, J., \& Kuipers, P. (2006). A metacognitive contextual intervention to enhance error awareness and functional outcome following traumatic brain injury: A single-case experimental design. Journal of the International Neuropsychological Society, 12(1), 5463.

Ownsworth, T., McFarland, K., \& Young, R.M. (2000). Self-awareness and psychosocial functioning following acquired brain injury: An evaluation of a group support programme. Neuropsychological Rehabilitation, 10, 465-484.

Penn, D.L., Corrigan, P.W., Bentall, R.P., Racenstein, J. M., \& Newman, L. (1997). Social cognition in schizophrenia. Psychological Bulletin, 121, 114132.

Poveda, B., Laidlaw, K., Macleod, F., Power, K., \& Osborne-Crowley, K. (2017). Social cognition, behaviour and relationship continuity in dementia of the Alzheimer type. Brain Impairment.

Rankin, K.P., Kramer, J.H., \& Miller, B.L. (2005). Patterns of cognitive and emotional empathy in frontotemporal lobar degeneration. Cognitive and Behavioral Neurology, 18(1), 28-36.

Roelofs, R.L., Wingbermühle, E., Egger, J.I.M., \& Kessels, R. P. C. (2017). Social cognitive interventions in neuropsychiatric patients: A meta-analysis. Brain Impairment, 18(1), 138-173.

Rushby, J., McDonald, S., Fisher, A.C., Kornfeld, E.J., De Blasio, F.M., Parks, N., \& Piguet, O. (2016). Brain volume loss contributes to arousal and empathy dysregulation following severe traumatic brain injury. Neuroimage: Clinical, 12, 607-614. Retrieved from: https://doi.org/10.1016/j.nicl.2016.09. 017

Ryan, N.P., Mihaljevic, K., Beauchamp, M.H., Catroppa, C., Crossley, L., Hearps, S., ... Anderson, , V.A. (2017). A prospective study of the relationship between family affective responsiveness and theory of mind in chronic paediatric traumatic brain injury. Brain Impairment, 18(1), 88-101.

Savla, G.N., Vella, L., Armstrong, C.C., Penn, D.L., \& Twamley, E.W. (2013). Deficits in domains of social cognition in schizophrenia: A meta-analysis of the empirical evidence. Schizophrenia Bulletin, 39(5), 979-992.

Silver, C.H. (2000). Ecological validity of neuropsychological assessment in childhood traumatic brain injury. Journal of Head Trauma Rehabilitation, 15(4), 973-988.

Turkstra, L.S., Kraning, S.G., Riedeman, S.K., Mutlu, B., Duff, M., \& VanDenHeuve, S. (2017). Labeling facial affect in context in adults with and without TBI. Brain Impairment, 18(1), 49-61.

Watts, A.J., \& Douglas, J.M. (2006). Interpreting facial expression and communication competence following severe traumatic brain injury. Aphasiology, 20(8), 707-722.

Wiig, E., \& Secord, W. (2014). Clinical evaluation of language fundamentals. USA: Pearson Assessment.

Wood, R.L., \& Williams, C. (2008). Inability to empathize following traumatic brain injury. Journal 
of the International Neuropsychological Society, 14, 289-296. Retrieved from: http://org/10.1017/ S1355617708080326

Yeates, K.O., Bigler, E.D., Dennis, M., Gerhardt, C.A., Rubin, K.H., Stancin, T., ... Vannatta, K. (2007). Social outcomes in childhood brain disorder: A heuristic integration of social neuroscience and developmental psychology. Psychological Bulletin, 133(3), 535-556. doi: 10.1037/0033-2909.133.3.535.

Yeates, K.O., Swift, E., Taylor, H.G., Wade, S.L., Drotar, D., Stancin, T., \& Minich, N. (2004). Short- and longterm social outcomes following pediatric traumatic brain injury. Journal of the International Neuropsychological Society, 10(3), 412-426.

Zupan, B., Babbage, D.R., Neumann, D., \& Willer, B. (2017). Sex differences in emotion recognition and emotional inferencing following severe traumatic brain injury. Brain Impairment, 18(1), 36-48.

Skye McDonald School of Psychology University of New South Wales

Sydney Australia 